\title{
APPLICATION OF TIME COMPRESSION APPROXIMATION METHOD TO INCORPORATE MULTI-STORM INFILTRATION PROCESSES IN YAMANASHI HYDROLOGICAL MODEL FOR ARID AND SEMI-ARID REGIONS
}

\author{
Guoqiang WANG ${ }^{1}$, Iain STRUTHERS 2 , Prasantha HAPUARACHCHI ${ }^{3}$, Kuniyoshi \\ TAKEUCHI $^{4}$ and Hiroshi ISHIDAIRA ${ }^{5}$ \\ ${ }^{1}$ Student member of JSCE, Ph. D. Student, Dept. of Civil and Environmental Engineering, University of Yamanashi \\ (4-3-11, Takeda, Kofu, Yamanashi 400-8511, Japan) \\ ${ }^{2}$ Dr. of Eng., Dept. of Civil and Environmental Engineering, University of Yamanashi (4-3-11, Takeda, Kofu, \\ Yamanashi 400-8511, Japan) \\ ${ }^{3}$ Dr. of Eng., Dept. of Civil and Environmental Engineering, University of Yamanashi (4-3-11, Takeda, Kofu, \\ Yamanashi 400-8511, Japan) \\ ${ }^{4}$ Member of JSCE, Dr. of Eng., Professor, Dept. of Civil and Environmental Engineering, University of Yamanashi \\ (4-3-11, Takeda, Kofu, Yamanashi 400-8511, Japan) \\ ${ }^{5}$ Member of JSCE, Dr. of Eng., Associate Professor, Dept. of Civil and Environmental Engineering, University of \\ Yamanashi (4-3-11, Takeda, Kofu, Yamanashi 400-8511, Japan)
}

\begin{abstract}
An approach is presented to solve the Philip's equation with time compression approximation (TCA) for simulating infiltration under variable rainfall. This approach brings understanding into physical processes of infiltration and ponding during variable rainfall storms. In particular, we apply it into the Yamanashi Hydrological Model (YHyM) for analyzing the infiltration excess runoff in arid and semi-arid regions. The soil water redistribution is also included to simulate the soil moisture changing during the temporal gaps among storms. The modified YHyM was tested by a case study of Lushi River basin, China. Through comparisons of results from the modified YHyM with results from the unmodified YHyM, the presented TCA based infiltration model is proved to be able to improve the performance of YHyM in peak runoff calculation during variable rainfall multi-storms in arid and semi-arid regions.
\end{abstract}

Key Words : Infiltration, time compression approximation, redistribution, multi-storm, YHyM

\section{INTRODUCTION}

Many research works have been done on the storm-based infiltration and runoff simulation in arid or semi-arid regions. But most studies were focused on single storm event simulation, which leave an important temporal gap between storms, especially during extreme storms. As $\mathrm{Yu}$ et al. ${ }^{1)}$ explained although catastrophic floods can result from single storms of long-term wet conditions, they commonly arise from a succession of storms often occurring over weeks to months. Therefore, hydrologic technique should be developed for periods ranging from two storms to many storms.

In this study, the Yamanashi Hydrological Model $(\mathrm{YHyM})^{2)}$ is used as a rainfall-runoff model for multi-storm runoff simulation. YHyM is developed based on the concepts of TOPMODEL ${ }^{3)}$ and Muskingum-Cunge routing method ${ }^{2}$. So far the model has been widely applied to large river basins mostly in daily time steps. The infiltration excess runoff is hardly generated, thus it is neglected. However the infiltration excess plays a major role in short time interval storm runoff simulation. In order to complete both infiltration excess and saturation excess runoff generation mechanisms in the YHyM, 
we combine the time compression approximation (TCA) with Philip's infiltration equation and incorporate this TCA based infiltration model into the YHyM for infiltration excess runoff generation.

TCA is a hydrologic approximation method in infiltration and water balance modeling. The concept was first used in the context of infiltration by Sherman ${ }^{4}$. The utility of TCA in this study is that for a given initial moisture state, one can use a single characteristic capacity curve derived as the solution to the infiltration rate under flooded surface concentration boundary conditions to model the actual infiltration capacity, even if the boundary is governed by a combination of preponding and postponding conditions. In recent years, researchers have done some works on the point infiltration simulation with TCA and suggested different opinions on the exactness of TCA. Liu and Parlange $^{5)}$, Salvucci and Entekhabi ${ }^{6)}$ discussed the relation of TCA to one rigorously defined flux concentration relation and used the latter to demonstrate the validity of TCA. They found that the largest error of TCA in calculating cumulative infiltration is only $2.5 \%$. In this paper, we try to express an improved way of TCA to deal with the infiltration under variable rainfall.

The following sections include descriptions of the TCA method under two rainfall types, soil water redistribution method and their applications in YHyM for infiltration simulation in arid and semi-arid regions. A case study is also employed to show the performance of YHyM after modification.

\section{DESCRIPTION OF YHyM}

The Yamanashi Hydrological Model (YHyM) is a distributed hydrological model developed at the University of Yamanashi. The core model included in YHyM is BTOPMC model, which stands for the Block-wise use of TOPMODEL with MuskingumCunge method ${ }^{2}$. According to the concept of BTOPMC, the basin is divided into several blocks or subbasins considering of the basin's scale and the heterogeneity of basin's topography. In the BTOPMC model, runoff generation is based on the TOPMODEL, which assumes the ground water flow is driven by the surface topographic gradient. YHyM has the advantages of both lumped and distributed models. It has relatively few parameters requiring calibration. The parameters for the model have physical interpretations, representing the effects of topography, vegetation $\left(S r_{\max }\right)$, soil properties $\left(T_{0}, M, n_{0}\right)$. YHyM is also a GIS based model, which abstracts the spatial data from DEM and relates parameters to basin features.
Like TOPMODEL, YHyM is a saturation excess based hydrological model. Therefore in wet regions, it performs well, but in arid and semi-arid regions, the performance is poor. To solve this problem, it is necessary to incorporate a simple infiltration method preferably with few parameters, though improving the performance of the YHyM.

\section{TCA BASED INFILTRATION MODEL}

Time compression approximation is derived from the assumption that maximum capacity at which a soil column can infiltrate at any given time during storms depends only on the initial moisture state of the column and the cumulative infiltration up to that time. Under this assumption, for given the initial soil conditions, the storm infiltration capacity $f(t)$ can be expressed as a function of cumulative storm infiltration $F(t)$ by TCA after the ponding time.

$$
f(t)=G_{T C A}(F(t))
$$

The actual infiltration rate during the event $f^{*}(t)$ is the lesser of potential infiltration capacity and the rainfall intensity $p(t)$.

$$
f^{*}(t)=\operatorname{Min}(f(t), p(t))
$$

In this study, Philip's equation is selected as the basic infiltration equation and combined with TCA. Philip $^{7)}$ represented the infiltration equation as:

$$
\begin{aligned}
f(t) & =\frac{1}{2} S t^{-1 / 2}+\frac{2}{3} K_{s} \\
\text { or } \quad F(t) & =S t^{1 / 2}+\frac{2}{3} K_{s} t
\end{aligned}
$$

where $S$ is the soil sorptivity, which depends on the soil water potential (in following sections, $S$ denotes the initial sorptivity), $K_{s}$ is the saturated hydraulic conductivity. $S$ can be derived from the equation:

$$
\left[S\left(\theta_{i}\right)\right]^{2}=2\left(\theta_{s}-\theta_{i}\right) \int_{\theta_{i}}^{\theta_{s}} K(\theta)\left(\frac{d \psi}{d \theta}\right) d \theta
$$

in which $\theta_{i}$ and $\theta_{s}$ are initial water content and saturated water content respectively.

The $K(\theta)$ in Eq. (4) is assumed to fit Brooks and Corey model ${ }^{8)}$ and $\psi(\theta)$ is got from Smith's ${ }^{9)}$ equation:

$$
\begin{gathered}
K(\theta)=K_{s}\left[\frac{\theta-\theta_{r}}{\theta_{s}-\theta_{r}}\right]^{b+(a / \lambda)} \\
\psi(\theta)=\psi_{b}\left[\left(\frac{\theta-\theta_{r}}{\theta_{s}-\theta_{r}}\right)^{-(c / \lambda)}-1\right]^{1 / c}
\end{gathered}
$$

where $\psi_{b}$ is the air entry potential, $\theta_{r}$ is the residual soil water content, $a, b, c$ and $\lambda$ are dimensionless coefficients.

Under TCA, the infiltration functions can be simplified into either time-based equation by 
eliminating the cumulative infiltration from the equations, or as a cumulative infiltration-based equation by solving out the time. In this study, the time-based TCA method is adopted.

\section{(1) Application of TCA under constant rainfall}

The TCA method is firstly introduced in the infiltration simulation under constant rainfall. Under TCA assumptions, both potential infiltration capacity and actual infiltration capacity during a storm can be defined by cumulative infiltration and initial soil condition.

By the definition of TCA, the actual infiltration capacity during storms will equal to the constant rainfall rate $p$ at the ponding time $t_{p}$.

$$
f^{*}\left(t_{p}\right)=p
$$

As to the potential infiltration capacity, TCA defines a compression time $t_{c}$ as the time when the potential infiltration capacity equals rainfall rate.

$$
f\left(t_{c}\right)=p
$$

By the TCA postulates, infiltration capacity is only dependent on cumulative infiltration and initial conditions. Thus with same initial conditions and infiltration rates, the potential infiltration rate and the actual infiltration rate will have same cumulative infiltration volumes at times of $t_{c}$ and $t_{p}$. Therefore the infiltration capacity will be identical after the time $t_{c}$ and $t_{p}$ for two situations.

$$
f^{*}\left(t_{p}+t\right)=f\left(t_{c}+t\right)
$$

Accordingly, from the start time at the beginning of a storm, the expression for actual infiltration capacity under the time compression approximation (illustrated in Fig.1) can be represented as:

$$
\begin{array}{ll}
f^{*}(t)=p & \text { for } t<t_{p} \\
f^{*}(t)=f\left(t-\left(t_{p}-t_{c}\right)\right) & \text { for } \quad t \geq t_{p}
\end{array}
$$

The compression time $t_{c}$ can be solved with Eq. (8) and Eq. (3a) ( $A_{0}$ is used to represent $\frac{2}{3} K_{S}$ ).

$$
t_{c}=\frac{S^{2}}{4\left(p-A_{0}\right)^{2}}
$$

Before the ponding time, the infiltration rate is dominated by rainfall, so the cumulative infiltration for actual storm infiltration capacity before ponding time equals the cumulative rainfall.

$$
F^{*}\left(t_{p}\right)=F\left(t_{c}\right)=p \cdot t_{p}
$$

Thus the ponding time $t_{p}$ can be solved as:

$$
t_{p}=\frac{S^{2}}{4 p A_{0}}\left[\frac{p^{2}}{\left(p-A_{0}\right)^{2}}-1\right]
$$

\section{(2) Application of TCA under variable rainfall}

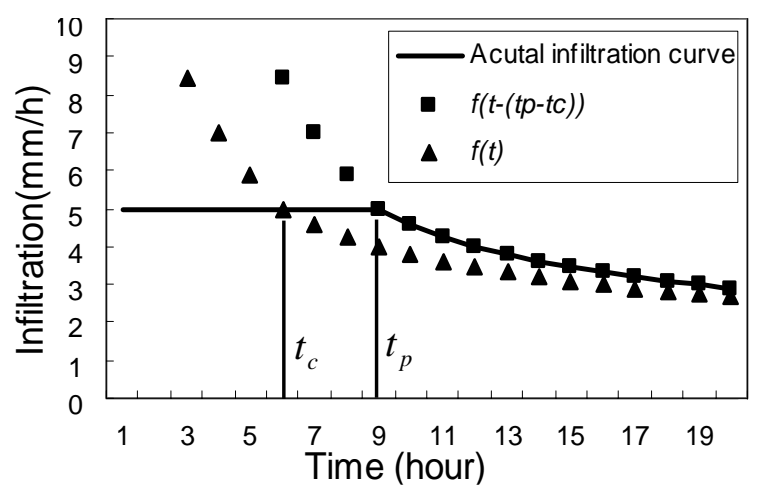

Fig.1 Illustration of TCA for infiltration under constant rainfall

Consider the rainfall defined during storm duration with within-event variability in rainfall intensity at any given time resolution $\Delta t$ (i.e. rainfall intensity is constant within time step $\Delta t$, but variable between time steps). Under TCA, we should be able to use a similar approach, but for each time step we need to re-calculate $t_{c}$ and $t_{p}$ with stepwise variable rainfall $p_{i}$ ( $i$ denotes the time step). $t_{c, i}$ ( $t_{c}$ during time step $i$ ) can be obtained from Eq. (11) with different rainfall intensities, and $t_{p, i}$ ( $t_{p}$ during time step $i$ ) can be re-calculated for each time step as:

$$
t_{p, i}=\frac{S \cdot\left(t_{c, i}\right)^{1 / 2}+A_{0} \cdot t_{c, i}-F\left(t_{(i-1)}\right)}{p_{i}}
$$

$F\left(t_{(i-1)}\right)$ is the cumulative infiltration from start to the time step $i-1$ (for first time step, $F\left(t_{(i-1)}\right)=0$ ).

Under TCA, we are actually interested in cumulative infiltration rather than infiltration per time step, therefore the cumulative infiltration can be stated with the infiltration in each time step.

$$
\sum_{1}^{i} F\left(t_{i}-\left(t_{p, i}-t_{c, i}\right)\right)=\sum_{1}^{i} p_{i} \cdot \Delta t
$$

During variable rainfall multi-storms, for each new storm, it is necessary to re-calculate $S$ according to the initial soil water content based on cumulative infiltration, evaporative loss and soil water redistribution after the preceding storm. But within a given storm, the rainfall is changing with time steps, thus the infiltration capacity can not be simulated continuously. If rainfall is greater than the initial infiltration capacity at the start of a new time step, the ponding time will be negative $\left(t_{p, i}<0\right)$. In order to counteract the stepwise rainfall variability within one storm, we introduced an offset time $t_{f, i}$ into the TCA structure. Within one storm, the infiltration capacity at the start of time step $i+1$ always equals the infiltration capacity at the end of time step $i$, thus the offset time is represented as:

$$
f^{*}\left(t_{i+1}+t_{f, i}\right)=f\left(t_{i}-\left(t_{p, i}-t_{c, i}\right)\right)
$$


Combing Eq. (16), Eq. (3a), Eq. (11) and Eq. (14) together, the offset time can be solved as:

$$
t_{f, i}=\frac{2 A_{0} F\left(t_{(i-1)}\right)+S^{2}-S \sqrt{4 A_{0} F\left(t_{(i-1)}\right)+S^{2}}}{2 A_{0}^{2}}
$$

In a summary, under stepwise variable rainfall, if $\Delta t \leq t_{p, i}$, no ponding occurs within the time step, if $\Delta t>t_{p, i}$, ponding occurs, under this situation, there are two possible solutions depending on whether $t_{p, i}<0$. All the three possible expressions can be summarized as function of the infiltration volume during one given time step.

$$
\begin{array}{r}
\Delta F\left(t_{i}\right)=p_{i} \cdot \Delta t \quad \text { if } \quad p_{i} \leq A_{0} \\
\begin{aligned}
\Delta F\left(t_{i}\right) & =A_{0} \cdot \Delta t+S \cdot\left(\sqrt{\Delta t+t_{f, i}}-\sqrt{t_{f, i}}\right) \\
& \text { if } \quad p_{i}>A_{0}, t_{p, i}<0
\end{aligned} \\
\begin{aligned}
& \Delta F\left(t_{i}\right)=p_{i} \cdot t_{p, i}+A_{0} \cdot\left(\Delta t-t_{p, i}\right)+S \\
&\left(\sqrt{\Delta t-\left(t_{p, i}-t_{c, i}\right)}-\sqrt{t_{c, i}}\right) \\
& \text { if } \quad p_{i}>A_{0}, t_{p, i} \geq 0
\end{aligned}
\end{array}
$$

In the field, the rainfall intensity can vary thus it is hard to measure the actual infiltration rate but not for the cumulative infiltration. Using TCA, the infiltration capacity can be easily calculated with reasonable accuracy.

Though the TCA method emphasizes the cumulative infiltration, it still keeps the dependence on the initial soil properties. Thus the role of initial soil conditions in governing the potential for runoff is considered in this study. Usually, under conditions of high initial soil water content, the potential infiltration capacity is lower and the ponding time is short, thus the infiltration excess runoff can be enhanced by increasing the parameter of initial soil moisture.

\section{SOIL WATER REDISTRIBUTION}

The term redistribution generally refers to the movement of soil moisture induced by lack of equilibrium within the profile, and in particular refers to the downward movement of water following infiltration.

After a storm, redistribution first occurs at high rates, but it quickly decelerates as the hydraulic conductivity and the head gradients decrease. The net result is further penetration of the infiltration wave and reduction of the volumetric moisture content especially near the soil surface.

If there are obvious gaps among multi-storms, when the rainfall rate falls below $K_{s}$ or equals zero, the soil water redistributes, with surface $\theta$ falling below $\theta_{s}$. During redistribution, the water content in the soil is expected to be characterized by a smooth but time-varying profile, particularly for moderately long redistribution periods. Such a profile maybe adequately approximated by following the similarity in shape for estimation of the $\theta_{i}$ for a subsequent storm event.

Chen et al. ${ }^{10)}$ researched on the redistribution of soil moisture during several continuous storms in Yellow River basin and found that the soil moisture in each layer responds differently to a rainfall event, and it may show little or no change due to antecedent storage conditions, but there is a large increase in response to continuous storms. Therefore soil water redistribution is incorporated to complete the infiltration simulation during multi-storms.

According to Smith's research ${ }^{9)}$, the evolution equation of surface soil water content $\theta_{0}$ can be expressed as:

$$
\begin{aligned}
\frac{d \theta_{0}}{d t}= & \frac{\theta_{0}-\theta_{i}}{f(t)}\left[p_{i}-K\left(\theta_{i}\right)-\left(K\left(\theta_{0}\right)+\right.\right. \\
& \left.\left.\frac{\beta \cdot \rho \cdot K_{s} \cdot\left(\theta_{0}-\theta_{i}\right) \cdot G\left(\theta_{i}, \theta_{0}\right)}{f(t)}\right)\right]
\end{aligned}
$$

where $\rho$ is shape factor approaching 1 when rainfall $p$ gets near $K_{s} . \quad \beta$ is a constant scale factor which is defined as:

$$
\beta=\frac{1}{Z\left(\theta_{0}-\theta_{i}\right)} \int_{0}^{z}\left(\theta-\theta_{i}\right) d z
$$

$Z$ in Eq. (20) is the depth of redistribution zone.

$G$ used in Eq. (16) is an integral capillary drive value derived from the following general function:

$$
G\left(\theta_{i}, \theta_{0}\right)=\frac{1}{K_{s}} \int_{\theta_{i}}^{\theta_{0}} K(\theta)\left(\frac{d \psi}{d \theta}\right) d \theta
$$

In a summary, during multi-storm gaps, the soil moisture changes with the soil water redistribution. Therefore the model for redistribution is included to calculate the soil moisture changing and initialize the infiltration parameters for the next storms. The soil water redistribution will end if $p_{i}>K_{s}$.

\section{CASE STUDY}

A case study is undertaken in the Lushi River basin to justify the validity of the modified YHyM.

\section{(1) Study area}

Lushi River basin is located southeast of Yellow River basin in China $\left(109^{\circ} 40^{\prime} \sim 110^{\circ} 10^{\prime} \mathrm{E}, 33^{\circ} 45^{\prime}\right.$ $\sim 34^{\circ} 30^{\prime} \mathrm{N}$ ). The basin has an area of $4623 \mathrm{~km}^{2}$ and length of $196.3 \mathrm{~km}$. Lushi River basin belongs to the semi-arid region with mean annual precipitation of $720 \mathrm{~mm}$ and potential evaporation of $966 \mathrm{~mm}$. The topographical characteristics of the basin were derived from a $100 \mathrm{~m}$ digital elevation model. The 


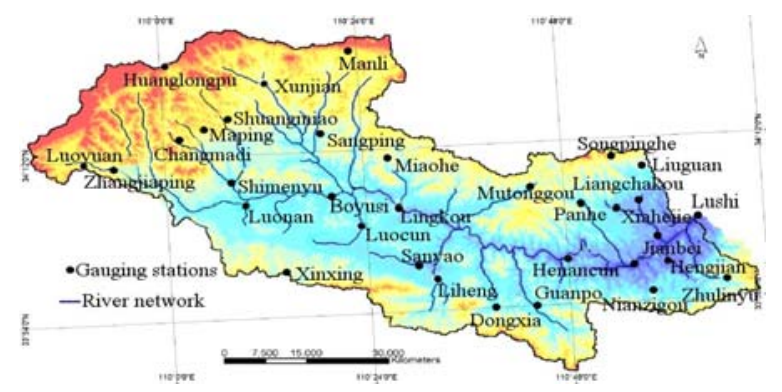

Fig.2 Map of rainfall gauging stations in Lushi River basin

Table 1 Initial infiltration parameters for dominant soil types

\begin{tabular}{|c|c|c|}
\hline Parameters & Silty clay & Sandy clay \\
\hline \hline$K_{S}(\mathrm{~mm} / \mathrm{h})$ & 0.5 & 0.6 \\
\hline$\theta_{S}$ & 0.479 & 0.432 \\
\hline$\theta_{i}$ & 0.335 & 0.325 \\
\hline$\theta_{r}$ & 0.123 & 0.121 \\
\hline$\psi_{b}(\mathrm{~mm})$ & -25 & -25 \\
\hline$\lambda$ & 0.2 & 0.2 \\
\hline$a$ & 2 & 2 \\
\hline$b$ & 3 & 3 \\
\hline$c$ & 5 & 5 \\
\hline$G\left(\theta_{\mathrm{i}}\right)(\mathrm{mm})$ & 292.2 & 239.1 \\
\hline
\end{tabular}

hourly rainfall from 33 gauging stations (see Fig.2) and runoff data of Lushi station from the year 1960 to 1997 are available for this simulation.

\section{(2) Initial values of infiltration parameters}

Using TCA concept, the difficulties caused by considering temporal variation of soil moisture can be avoided; however, the quantification of the initial infiltration parameters becomes important for the model's performance. There are 12 kinds of soil types in the Lushi basin. The infiltration parameters for each soil type are initialized based on the soil physical properties ${ }^{9), 11)}$ and finely tuned. Table 1 only shows the infiltration parameters for two dominant soil types in the Lushi basin.

\section{(3) Simulation results}

In this case study, the modified YHyM is tested for the multi-storm runoff simulations. Rainfall data for 7 storms in 3 years are selected, that is, 3 storms in 1988, 2 storms in 1992 and 2 storms in 1994. In order to justify the validity of the infiltration model used, we compare the runoff results from the unmodified YHyM and the modified YHyM. The multi-storm hydrographs of Lushi station in these years from both models are shown in Fig.3 to Fig.8. In this study, the parameters of the new infiltration model in the YHyM were tuned using the data of 1988 and validated using the data of the other two years. The runoff simulation results from the

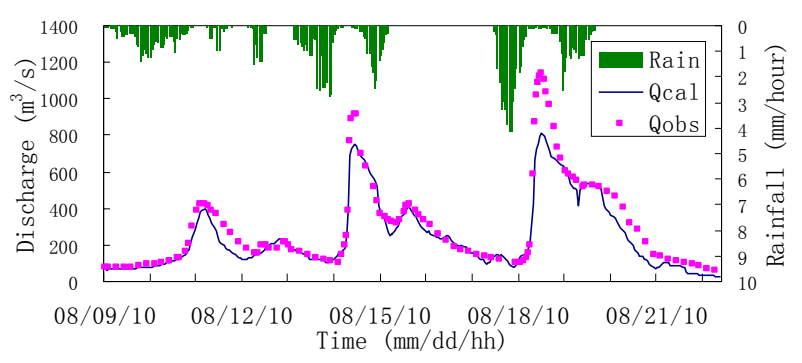

Fig.3 Multi-storm hydrograph from unmodified YHyM (Lushi station - 1988).

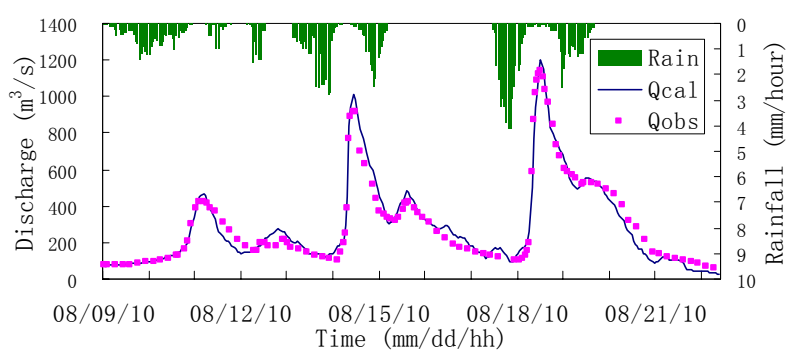

Fig.4 Multi-storm hydrograph from modified YHyM (Lushi station - 1988).

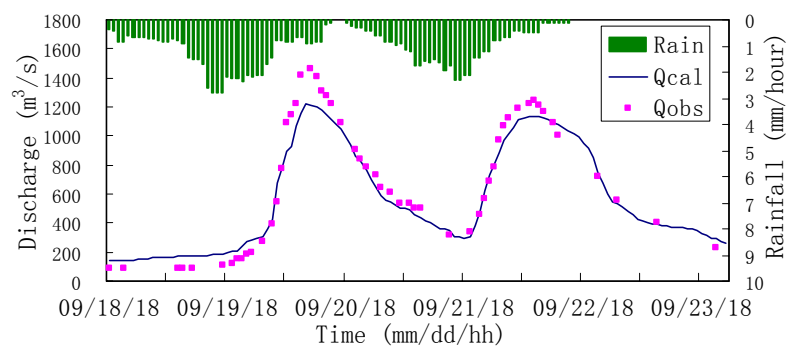

Fig.5 Multi-storm hydrograph from unmodified YHyM (Lushi station - 1992).

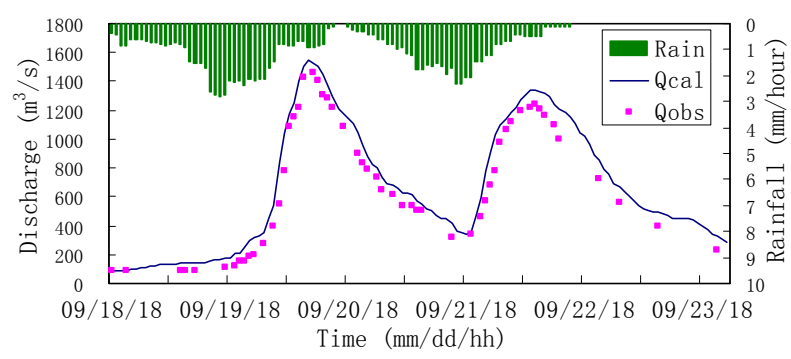

Fig.6 Multi-storm hydrograph from modified YHyM (Lushi station - 1992).

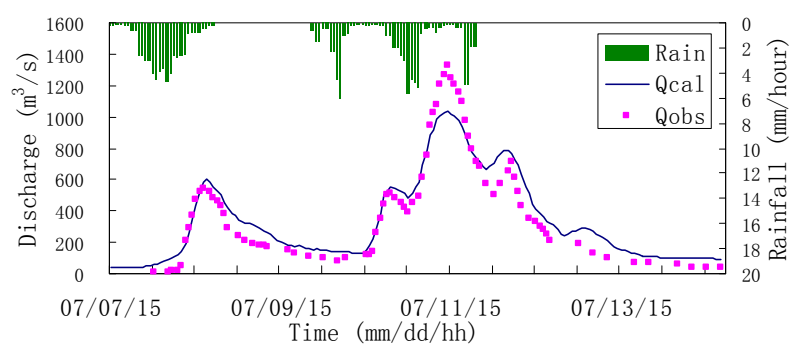

Fig.7 Multi-storm hydrograph from unmodified YHyM (Lushi station - 1994). 


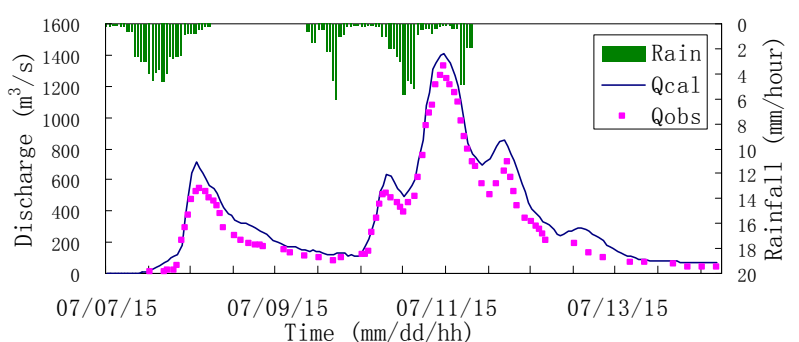

Fig.8 Multi-storm hydrograph from modified YHyM (Lushi station - 1994).

modified YHyM show good coherence with the observed data in all these 3 years, especially, for the peak runoff results. During the simulations, the common hydrological parameters for both YHyM versions share the identical values.

On the whole, the simulation results from the modified YHyM with infiltration excess are in better accordance with the observed data of Lushi station compared to the unmodified YHyM. The Nash efficiencies for the results from the modified YHyM are 93.8\%, 92.8\% and 93.1\% and for the results from the unmodified YHyM are $86.4 \%$, 78.7\% and 88.1\% in the year 1988, 1992 and 1994 respectively. Therefore the TCA based infiltration model is proved to be able to improve the performance of YHyM in storm runoff simulation, especially for the peak runoff simulation. However, during low runoff simulations, the modified YHyM does not show obvious advantages.

\section{CONCLUSION}

Time compression approximation provides an adequate tool for description transition of unstable, state dependent infiltration processes from rainfall control to soil control. Based on the previous researchers' works, this study focused on improving the performance of TCA in infiltration calculation under stepwise variable rainfall for arid or semi-arid river basins and proving its validity in the YHyM for runoff calculation in large regions.

For multi-storm events, there are some obvious temporal gaps among the storms. During these temporal gaps, the soil moisture changes. In order to process the successive evolution of infiltration accurately during continuous storms, the soil water redistribution model is included into the TCA structure to bridge these gaps. Thus, the initial soil moisture at the start time of the next storm can be initialized accurately by re-calculating sorptivity $S$ according to the soil moisture changing during redistribution period from the preceding storm.

In this study, the Philip's equation is selected to be coupled with TCA, because of its physical basis and simple form. This TCA based infiltration model is coupled into YHyM to incorporate the infiltration excess runoff mechanism in the model. Through the case study of Lushi River basin, the modified TCA method is found to be capable of improving the performance of YHyM in calculating the peak runoff while keeping high computation efficiency.

YHyM is a powerful hydrological tool for large river basin simulation. While in a large region, numbers of soil types will decrease the calculation efficiency. YHyM can combine all soil types into several dominant types and initialize the mean parameters for each type. The TCA method appears to fit this initialization quite well.

ACKNOWLEDGMENT: The authors gratefully acknowledge the MEXT, "Grant-in-aid for scientific research: Downscaling of global observations into local scale information”, University of Yamanashi 21st COE program.

\section{REFERENCES}

1) Yu, Z., Barron, E.J. and Yarnal, B.: Evaluation of basin-scale hydrologic response to a multi-storm simulation, J. Hydrol., Vol.257, pp.212-225, 2002.

2) Ao, T., Takeuchi, K. and Ishidaira, H.: On problems and solutions of the Muskingum-Cunge routing method applied to a distributed rainfall runoff model (in Japanese), Annual J. Hydraulic Engg., JSCE, Vol.44, pp.139-144, 2000.

3) Beven, K.J. and Kirkby, M.J.: A physically based, variable contributing area model of hydrology, Hydrol. Sci. Bul., Vol.24(1), pp.43-69, 1979.

4) Sherman, L.K.: Comparison of F-curves derived by the methods of Sharp and Holtan and of Sherman and Mayer, Eos. Trans. AGU., Vol.24, pp.465-467, 1943.

5) Liu, M.C., Parlange, J.Y., Sivapalan, M. and Brutsaert, W.: A note on the time compression approximation, Water Resour. Res., Vol.34(12), pp.3683-3686, 1998.

6) Salvucci, G.D. and Entekhabi, D.: Equivalent steady soil moisture profile and the time compression approximation in water balance modeling, Water Resour. Res., Vol.30(10), pp.2737-2749, 1994.

7) Philip, J.R.: The theory of infiltration: 1 . The infiltration equation and its solution, Soil Sci., Vol.83, pp.345-357, 1957.

8) Brooks, K. and Corey, A.: Hydraulic properties of porous media. Hydrology paper No.3, Colorado State University Press, 1964.

9) Smith, R.E., Corradini, C. and Melone, F.: Modeling infiltration for multistorm runoff events, Water Resour. Res., Vol.29(1), pp.133-144, 1993.

10) Chen, J.Y., Tang, C.Y. and Sakura, Y.: Measurement and analysis of the redistribution of soil moisture and solutes in a maize field in the lower reaches of the Yellow River, Hydrol. Process., Vol.18, pp.2263-2273, 2004.

11) Hogentogler, C.A.: Engineering properties of soil, McGraw-Hill Book Company, 1937.

(Received September 30,2005) 